\title{
Magnetically coupled quadrature all-pass filter for quadrature signal generation in vector-summing phase shifters
}

\author{
Chia-Wei $\mathrm{Hsu}^{1}$ and Jia-Shiang Fu ${ }^{1 \mathrm{a})}$
}

\begin{abstract}
For a vector-summing phase shifter, the quadrature signal generation (QSG) network is crucial. Among the existing QSG networks, a one-stage quadrature all-pass filter (QAF) provides the same bandwidth as a two-stage $R-C$ polyphase filter while exhibiting 6-dB higher voltage gain. Despite all the advantages, the major disadvantage of the conventional QAF is that it contains two inductors, which occupy large chip area. In this work, magnetically coupled QAF (MCQAF) is proposed. In the proposed MCQAF, the two inductors in the QAF are intertwined to reduce the occupied chip area, dispelling the major disadvantage of the conventional QAF. With the magnetic coupling between the inductors considered, the design equation for the inductance in the MCQAF is derived. An MCQAF is designed at $2 \mathrm{GHz}$ in a GaAs integrated passive device process along with a conventional QAF for comparison. The results of this work show that, except for a slightly larger amplitude error, the proposed MCQAF exhibits performances comparable to those of the conventional QAF while the occupied chip area is reduced by approximately $1 / 3$.

key words: quadrature signal generation, quadrature all-pass filter, magnetic coupling, phase shifter, vector summing

Classification: Integrated circuits (RF)
\end{abstract}

\section{Introduction}

Phased arrays are important for many applications, such as fifth-generation $(5 \mathrm{G})$ mobile communication, radar, and satellite communication. Phase shifters are essential components in phased-array transceivers. Among various circuit topologies, the vector-summing architecture [1-18] is extensively used for the design of active phase shifters. The block diagram and operating principle of a generic vectorsumming phase shifter are shown in Fig. 1(a) and (b), respectively. As shown in Fig. 1(a), first of all, a quadrature signal generation (QSG) network is required to produce inphase (I) and quadrature $(\mathrm{Q})$ signals, which are $90^{\circ}$ out of phase. Next, the I and Q signals are separately amplified by variable-gain amplifiers (VGAs) and then summed up. Fig. 1(b) illustrates that, on the I-Q plane, the output signal is the vector sum of the amplified I and Q signals. Consequently, the phase of the output signal is determined by the ratio of the gains of the VGAs in the I and Q paths. Once the

\footnotetext{
${ }^{1}$ Dept. of Electrical Engineering, National Central University, Taoyuan, Taiwan

a) jsfu@ee.ncu.edu.tw
}

DOI: $10.1587 /$ elex.18.20210330

Received August 08, 2021

Accepted August 25, 2021

Publicized September 01, 2021
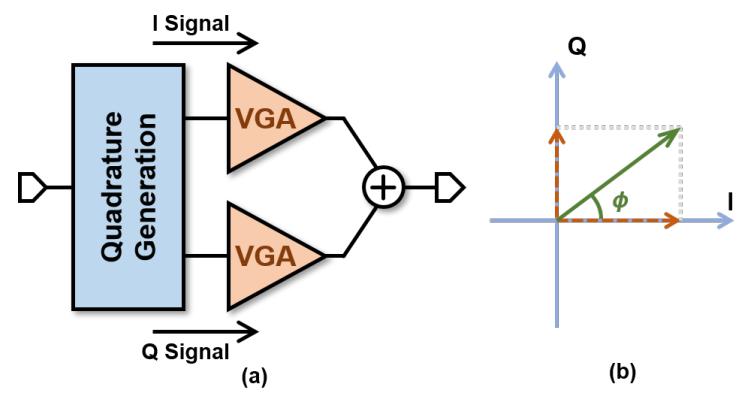

Fig. 1. (a) Block diagram and (b) operating principle of vector-summing phase shifter.

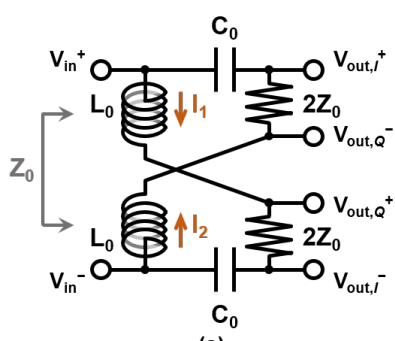

(a)

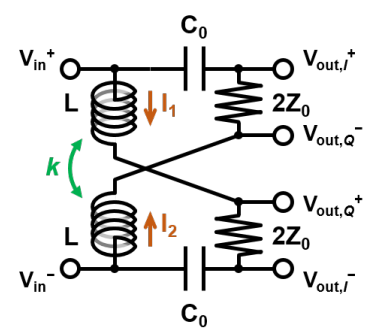

(b)
Fig. 2. Schematics of (a) the conventional QAF and (b) the proposed magnetically coupled QAF (MCQAF).

gain ratio varies from one value to another, the phase of the output signal is shifted. For a vector-summing phase shifter, the QSG network is crucial. If the phase difference between the two output signals of the QSG network deviates from $90^{\circ}$, the accuracy of phase shift gets degraded and the phase error of the phase shifter subsequently increases. As a result, the phase bandwidth of a vector-summing phase shifter is limited by the bandwidth of its QSG network.

The $R-C$ polyphase filter (PPF) $[19,20]$ has been widely adopted for quadrature signal generation. The $R-C$ PPF is used extensively in mixers and in vector-summing phase shifters, too. The advantage of $R-C$ PPF is that, since it is made of resistors and capacitors, it occupies relatively small chip area. However, one-stage $R-C$ PPF is narrowband and has a voltage gain of only $0 \mathrm{~dB}$. To obtain a wider bandwidth, two-stage $R-C$ PPF is usually necessary. In spite of the increased bandwidth, a two-stage $R-C$ PPF provides a even lower voltage gain of $-3 \mathrm{~dB}$, which, in the context of active phase shifter design, implies that more DC power is required 
if the lowered gain should be recovered. To avoid the loss incurred by $R-C \mathrm{PPF}$, quadrature all-pass filter (QAF), as depicted in Fig. 2(a), was proposed [21]. A one-stage QAF has the same bandwidth as a two-stage $R-C$ PPF while providing 6-dB higher voltage gain [22]. Despite all the advantages, the major disadvantage of the conventional QAF is that it contains two inductors, which occupy large chip area.

When a QAF is implemented in integrated circuit form, its two inductors may be in close proximity to each other, which results in magnetic coupling between the two inductors. In this work, magnetically coupled QAF (MCQAF), shown in Fig. 2(b), is proposed. In the proposed MCQAF, the two inductors in the QAF are deliberately intertwined to form a transformer. As a result, the occupied chip area is reduced, dispelling the major disadvantage of the conventional QAF. Similar yet different QSG circuit topologies that also use transformers exist in the literature [23-29]. Both the transformer-based quadrature hybrid [23-25] and the transformer-based polyphase network [26-29] originated from the concept of realizing the coupled-line coupler with lumped elements [30,31]. On the other hand, the QAF was invented by replacing the branches in the $R C-C R$ QSG network with series $R L C$ resonators [21,22]. Evolving from the conventional QAF, the proposed MCQAF is therefore a new addition to the existing QSG networks.

Since magnetic coupling is present between the two inductors in the MCQAF, the design equation for the inductance is therefore different from that of the conventional QAF. In the following text, the modified design equation is derived. Moreover, an MCQAF is designed at $2 \mathrm{GHz}$ in a GaAs integrated passive device (IPD) process along with a conventional QAF for comparison. Next, measurement results of the chip are presented. Finally, the conclusion is given.

\section{Design}

The schematic of the conventional QAF is shown in Fig. 2(a). The conventional QAF was analyzed in [21] and the nominal component values are as follows. The inductance

$$
L_{0}=\frac{Z_{0}}{\omega_{0}}
$$

and the capacitance

$$
C_{0}=\frac{1}{\omega_{0} Z_{0}},
$$

where $Z_{0}$ and $\omega_{0}$ are the system impedance and center frequency, respectively. The nominal value for the resistance is $2 Z_{0}$, which results in an exact $90^{\circ}$ phase difference between the output I and Q signals while achieving a perfect input match at $\omega_{0}$. The resistance could however be adjusted to a higher value, corresponding to a lower quality factor for the series $R L C$ resonance, to trade the phase accuracy at $\omega_{0}$ for a wider bandwidth [21]. Note that, just like $R-C$ PPF, in the ideal analysis of QAF, the output ports are assumed to be open-circuited. Nonetheless, the effects of capacitive loading have been considered in [21].

The schematic of the MCQAF is shown in Fig. 2(b). To obtain the design equations, one may analyze the circuit using KCL and KVL. Nevertheless, by comparing the two circuits shown in Fig. 2(a) and (b), it can be inferred that the only difference is how the voltage across the inductor relates to the current flowing through it. As shown in Fig. 2(a), if the current flowing through the upper inductor, which is connected to the positive input terminal, is denoted by $I_{1}$, for the conventional QAF,

$$
V_{\text {in }}^{+}-V_{\text {out }, Q}^{+}=j \omega L_{0} I_{1} .
$$

On the other hand, for the MCQAF in Fig. 2(b), the voltage across the upper inductor depends not only on the current flowing through itself, $I_{1}$, but also on the current flowing through the other inductor, $I_{2}$. That is,

$$
\begin{aligned}
V_{\text {in }}^{+}-V_{\text {out }, Q}^{+} & =j \omega L I_{1}+j \omega M I_{2} \\
& =j \omega L I_{1}-j \omega M I_{1} \\
& =j \omega(L-M) I_{1} \\
& =j \omega(1-k) L I_{1},
\end{aligned}
$$

where $M$ is the mutual inductance between the two inductors and $k$ is the coupling coefficient. Note that $I_{2}=-I_{1}$ due to the symmetric nature of the structure. Comparing (4) with (3), it can be concluded that, if the MCQAF is to behave the same way as the conventional QAF does, the following condition must be met.

$$
L=\frac{L_{0}}{1-k}=\frac{1}{1-k} \frac{Z_{0}}{\omega_{0}} .
$$

To verify the proposed design, an MCQAF is designed at 2 $\mathrm{GHz}$ and implemented using a GaAs IPD process provided by WIN Semiconductors. Simplified cross section of the GaAs IPD process is shown in Fig. 3(a). The thickness of the GaAs substrate is $100 \mu \mathrm{m}$. The thickness of the gold metal layer on the backside of the substrate is $4.5 \mu \mathrm{m}$. On the front side, three gold metal layers (M1/M2/M3: 1/2/4 $\mu \mathrm{m})$ are available for interconnects. MIM capacitors with capacitance density up to $0.4 \mathrm{fF} / \mu^{2}$ and thin-film resistors with sheet resistance of $50 \Omega / \square$ are also available in the IPD process.

The layout of the MCQAF is shown in Fig. 3(b). Rectangular spiral inductors are used to realize the coupled inductors in the circuit. Except for the crossovers, the three metal layers are stacked together, with a total thickness of $7 \mu \mathrm{m}$, for the traces of the spiral inductors. The width of the traces and the gap between the adjacent traces are both $10 \mu \mathrm{m}$. For the crossovers, the upper traces are formed by the M3 layer whereas the lower traces are constructed by stacking the M1 and M2 layers. Note that, due to the symmetric nature of 


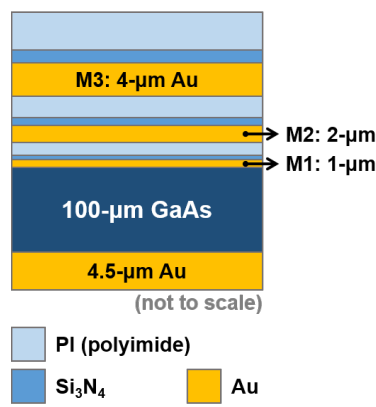

(a)

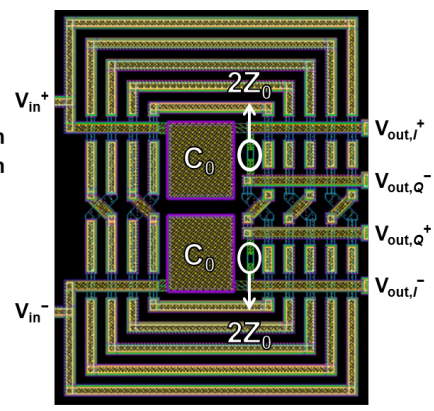

(b)
Fig. 3. (a) Simplified cross section of the GaAs IPD process and (b) the layout of the designed MCQAF.

Table I. Initial and final component values for the MCQAF $(k=-0.65)$ and the conventional QAF $(k=0)$.

\begin{tabular}{ccccc} 
& \multicolumn{2}{c}{$k=-0.65$} & \multicolumn{2}{c}{$k=0$} \\
\hline & Initial & Final & Initial & Final \\
\hline$L$ & $2.41 \mathrm{nH}$ & $2.29 \mathrm{nH}$ & $3.98 \mathrm{nH}$ & $3.53 \mathrm{nH}$ \\
$C$ & $1.59 \mathrm{pF}$ & $1.64 \mathrm{pF}$ & $1.59 \mathrm{pF}$ & $1.61 \mathrm{pF}$ \\
$R$ & $100 \Omega$ & $95.6 \Omega$ & $100 \Omega$ & $99.7 \Omega$ \\
\hline
\end{tabular}

this circuit and thereby the symmetric layout, the coupling coefficient $k$ is negative. By full-wave electromagnetic simulation, it is found that, with the $10-\mu \mathrm{m}$ width and gap, we are able to obtain a $k$ of -0.65 .

Using (5), the required inductance value is then calculated. On the other hand, the required capacitance value is calculated using (2). The system impedance $Z_{0}$ is $50 \Omega$ for this design. Component values, including the inductance, capacitance, and resistance, are then fine-tuned before the design is finalized. The actual component values used in the final design are summarized in Table I and compared against the initial theoretical values calculated using the design equations. Note that, for the purpose of comparison, a conventional QAF is designed at the same frequency in the same IPD process. The component values of the conventional QAF are listed in Table I as well.

Finally, it is worth mentioning that, with the way of layout shown in Fig. 3(b), we are able to place the capacitors and resistors inside the inductor loops, which makes the MCQAF more compact. Moreover, in our layout, the four outputs are on the same side and are across from the input port, which is most convenient for vector-summing phase shifters.

\section{Measurement Results}

The MCQAF is fabricated using the aforementioned WIN GaAs IPD process. The conventional QAF is fabricated on the same chip for comparison. The chip photo is shown in Fig. 4. The occupied areas of the conventional QAF and the MCQAF are $0.20 \times 0.77 \mathrm{~mm}^{2}\left(0.154 \mathrm{~mm}^{2}\right)$ and $0.28 \times 0.36$ $\mathrm{mm}^{2}\left(\sim 0.1 \mathrm{~mm}^{2}\right)$, respectively. By going from the conventional QAF to the MCQAF, the occupied area is reduced by approximately $1 / 3$.

As shown in the Fig. 4, additional crossovers and exten-

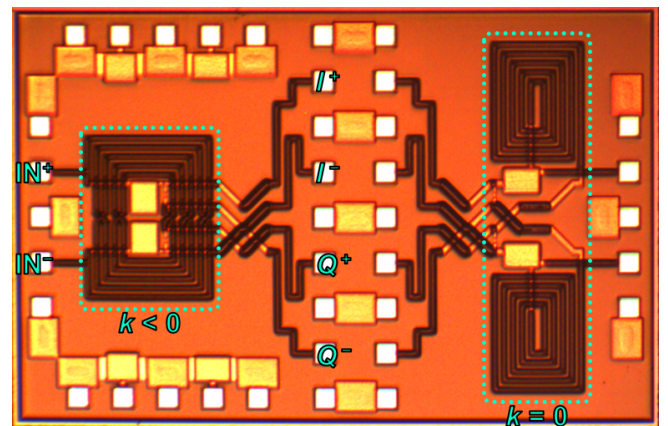

Fig. 4. Chip photo of the conventional QAF (right, $0.20 \times 0.77 \mathrm{~mm}^{2}$ ) and the MCQAF (left, $0.28 \times 0.36 \mathrm{~mm}^{2}$ ).

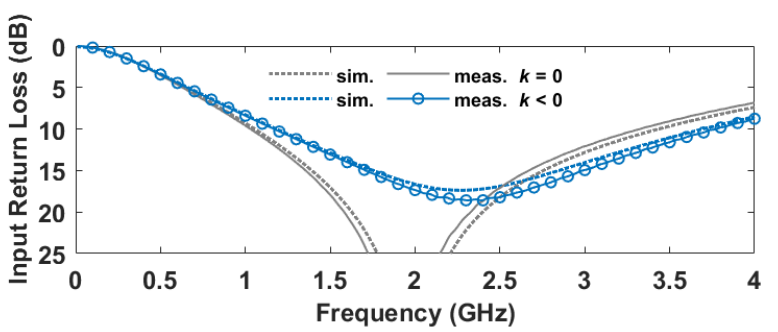

Fig. 5. Simulated (dotted) and measured (solid) input return losses of the conventional QAF $(k=0)$ and the MCQAF $(k<0)$.

sion lines are required at the output to pair the differential I/Q signals and direct them to the corresponding pads for probing. On-wafer measurement is performed with two GSGSG probes. One GSGSG probe is used at the input port while the other GSGSG probe contacts either the I output pads or the Q output pads. That is, the I and Q pads are probed in turn. Moreover, when one output port is connected, the other output port is left floating without further termination. Note that no termination is required because, ideally, the output terminals of a QAF should see open circuits. In this respect, QAF is in contrast with those QSG networks that originate from the quadrature hybrid [23-29], where the ports are terminated with the system impedance.

With the aforementioned way of probing, small-signal $S$ parameters of the QAFs are measured using a 4-port network analyzer (Keysight N5227B). Using Keysight ADS (Advanced Design System), AC simulations are then performed with the measured $S$ parameters to find the voltage gains and phase differences of the measured QAFs. The voltage gains are defined as follows.

$$
A_{V, I}=\left|\frac{V_{\mathrm{out}, I}^{+}-V_{\mathrm{out}, I}^{-}}{V_{\mathrm{in}}^{+}-V_{\mathrm{in}}^{-}}\right| \text {and } A_{V, Q}=\left|\frac{V_{\mathrm{out}, Q}^{+}-V_{\mathrm{out}, Q}^{-}}{V_{\mathrm{in}}^{+}-V_{\mathrm{in}}^{-}}\right| \text {. }
$$

The simulated and measured input return losses (with output ports open-circuited) of the conventional QAF and the MCQAF are shown in Fig. 5. Both QAFs exhibit $>15-\mathrm{dB}$ input return loss at $2 \mathrm{GHz}$. The simulated and measured voltage gains are plotted in Fig. 6. At $2 \mathrm{GHz}$, the voltage gains for both QAFs are close to the theoretical 3-dB value while the MCQAF shows larger imbalance between the magnitudes of its output I and Q signals. 


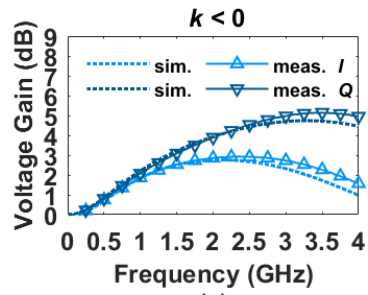

(a)

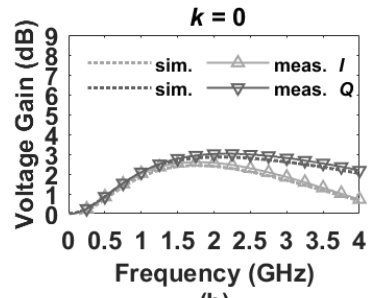

(b)
Fig. 6. Simulated (dotted) and measured (solid) voltage gains of (a) the $\operatorname{MCQAF}(k<0)$ and (b) the conventional QAF $(k=0)$.

In Fig. 7, the simulated and measured amplitude errors and phase errors of the QAFs are shown. The amplitude error (AE) is defined as the ratio of the magnitudes of the output I and Q signals, whereas the phase error (PE) is defined as the phase difference between the output I and Q signals subtracting the target $90^{\circ}$. At $2 \mathrm{GHz}$, the measured amplitude errors of the MCQAF and the conventional QAF are around $1.1 \mathrm{~dB}$ and $0.5 \mathrm{~dB}$, respectively. On the other hand, the measured peak phase errors of the MCQAF and the conventional QAF are about $5.6^{\circ}$ and $4.5^{\circ}$, respectively. Note that the phase error is intentionally made nonzero at 2 $\mathrm{GHz}$ for both designs to trade phase accuracy at the center frequency for wider bandwidth. This practice is called polesplitting technique in [21].

In summary, the measurement results show that, except for a larger amplitude error, the proposed MCQAF exhibits performances comparable to those of the conventional QAF. Through circuit simulation, it is found that the increased amplitude error is due to the parasitic capacitance, i.e., the electric coupling, between the two tightly intertwined inductors. The parasitic capacitance may be reduced by increasing the spacing between the windings of the two inductors.

\section{Conclusion}

In this work, a novel MCQAF is proposed for quadrature signal generation in vector-summing phase shifters. Compared to the conventional QAF, the two inductors of the proposed MCQAF are intertwined to reduce the occupied chip area. An MCQAF is designed at $2 \mathrm{GHz}$ and fabricated using a GaAs IPD process along with a conventional QAF. The results of this work show that, except for a slightly larger amplitude error, the MCQAF exhibits performances comparable to those of the conventional QAF while the occupied chip area is reduced by approximately $1 / 3$.

\section{Acknowledgments}

This work was supported in part by Ministry of Science and Technology, Taiwan, under Grant MOST 109-2221E-008-070, and in part by Qualcomm Technologies under Grant SOW NAT-435657 and Grant SOW NAT-457594. The authors thank Taiwan Semiconductor Research Institute for supporting chip fabrication. The authors thank National Center for High-Performance Computing of Taiwan

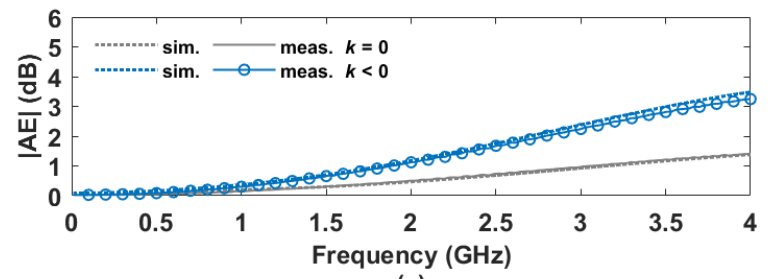

(a)

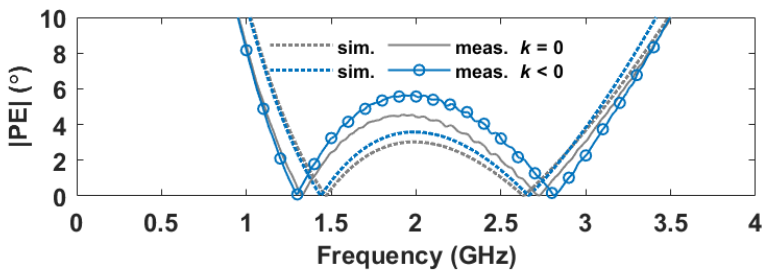

(b)

Fig. 7. Simulated (dotted) and measured (solid) absolute values of (a) amplitude errors and (b) phase errors of the conventional QAF $(k=0)$ and the MCQAF $(k<0)$.

for providing computational resources.

References

[1] Y. Zheng and C. E. Saavedra, "Full $360^{\circ}$ vector-sum phaseshifter for microwave system applications," IEEE Trans. Circuits Syst. I, vol.57, no.4, pp.752-758, April 2010.

[2] K.-J. Koh and G. M. Rebeiz, "A 6-18 GHz 5-bit active phase shifter," IEEE MTT-S Int. Microw. Symp. Dig., May 2010.

[3] T.-C. Yan, W.-Z. Lin, and C.-N. Kuo, "A 3.5-GHz phase shifter of high input power range with digitally controlled VGA,” Proc. IEEE Int. Symp. Radio-Freq. Integration Technol., Nov. 2011.

[4] Y.-Y. Huang, H. Jeon, Y. Yoon, W. Woo, C.-H. Lee, , and J. S. Kenney, "An ultra-compact, linearly-controlled variable phase shifter designed with a novel $R C$ poly-phase filter," IEEE Trans. Microw. Theory Techn., vol.60, no.2, pp.301310, Feb. 2012.

[5] A. Asoodeh and M. Atarodi, "A full $360^{\circ}$ vector-sum phase shifter with very low RMS phase error over a wide bandwidth," IEEE Trans. Microw. Theory Techn., vol.60, no.6, pp.1626-1634, June 2012.

[6] T.-C. Yan, W.-Z. Lin, and C.-N. Kuo, "A 0.75-2.67 GHz 5-bit vector-sum phase shifter," Proc. Euro. Microw. Integr. Circuits Conf., Oct. 2013.

[7] Z. Hu and K. Mouthaan, "A $0.5-6 \mathrm{GHz} 360^{\circ}$ vector-sum phase shifter in 0.13- $\mu \mathrm{m}$ CMOS," IEEE MTT-S Int. Microw. Symp. Dig., June 2014.

[8] E. V. Balashov and I. A. Rumyancev, "A fully integrated 6-bit vector-sum phase shifter in $0.18 \mu \mathrm{m}$ CMOS," Int. Siberian Conf. Control Commun., May 2015.

[9] B. Cetindogan, E. Ozeren, B. Ustundag, M. Kaynak, and Y. Gurbuz, "A 6 bit vector-sum phase shifter with a decoder based control circuit for X-band phased-arrays," IEEE Microw. Wireless Compon. Lett., vol.26, no.1, pp.64-66, Jan. 2016.

[10] M.-M. Mohsenpour and C. E. Saavedra, "Variable $360^{\circ}$ vector-sum phase shifter with coarse and fine vector scaling," IEEE Trans. Microw. Theory Techn., vol.64, no.7, pp.21132120, July 2016.

[11] M. Wang, Y. Liu, Z. Li, X. Wang, M. M. Sarfraz, Y. Xiao, and H. Zhang, "A 6-bit $38 \mathrm{GHz}$ SiGe BiCMOS phase shifter for $5 \mathrm{G}$ phased array communications," IEICE Electron. Ex- 
press, vol.14, no.13, June 2017.

[12] Z. Duan, Y. Wang, W. Lv, Y. Dai, and F. Lin, "A 6-bit CMOS active phase shifter for Ku-band phased arrays," IEEE Microw. Wireless Compon. Lett., vol.28, no.7, pp.615-617, July 2018.

[13] T. Fujiwara and M. Shimozawa, "Broadband and highly accurate X-band vector-sum phase shifter using LC-type power splitter," Proc. Euro. Microw. Integr. Circuits Conf., Sept. 2018.

[14] L. Luo, Z. Li, Y. Yao, and G. Cheng, "A 6-18 GHz 6bit active phase shifter in 0.13- $\mu$ m SiGe BiCMOS," IEICE Electron. Express, vol.16, no.10, April 2019.

[15] J. Xia and S. Boumaiza, "Digitally assisted $28 \mathrm{GHz}$ active phase shifter with $0.1 \mathrm{~dB} / 0.5^{\circ} \mathrm{RMS}$ magnitude/phase errors and enhanced linearity," IEEE Trans. Circuits Syst. II, vol.66, no.6, pp.914-918, June 2019.

[16] A. Hirai, T. Fujiwara, M. Tsuru, K. Mori, and M. Shimozawa, "Vector-sum phase shifter using a tunable active $g_{m}-C$ polyphase filter," IEEE Trans. Microw. Theory Techn., vol.68, no.10, pp.4091-4102, Oct. 2020.

[17] G. H. Park, C. W. Byeon, and C. S. Park, "A 60-GHz lowpower active phase shifter with impedance-invariant vector modulation in 65-nm CMOS," IEEE Trans. Microw. Theory Techn., vol.68, no.12, pp.5395-5407, Dec. 2020.

[18] F. Qiu, H. Zhu, L. Wu, W. Che, and Q. Xue, "A 15-38 $\mathrm{GHz}$ vector-summing phase-shifter with $360^{\circ}$ phase-shifting range using improved I/Q generator," IEEE Trans. Circuits Syst. II, April 2021.

[19] F. Behbahani, Y. Kishigami, J. Leete, and A. A. Abidi, "CMOS mixers and polyphase filters for large image rejection,” IEEE J. Solid-State Circuits, vol.36, no.6, pp.873-887, June 2001.

[20] J. Kaukovuori, K. Stadius, J. Ryynänen, and K. A. I. Halonen, "Analysis and design of passive polyphase filters," IEEE Trans. Circuits Syst. I, vol.55, no.10, pp.3023-3037, Nov. 2008.

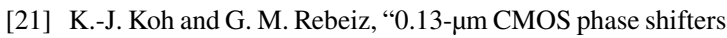
for X-, Ku-, and K-band phased arrays," IEEE J. Solid-State Circuits, vol.42, no.11, pp.2535-2546, Nov. 2007.

[22] S. Y. Kim, D.-W. Kang, K.-J. Koh, and G. M. Rebeiz, "An improved wideband all-pass I/Q network for millimeter-wave phase shifters," IEEE Trans. Microw. Theory Techn., vol.60, no.11, pp.3431-3439, Nov. 2012.

[23] C.-C. Chang, T.-Y. Chin, J.-C. Wu, and S.-F. Chang, "Novel design of a 2.5-GHz fully integrated CMOS Butler matrix for smart-antenna systems," vol.56, no.8, pp.1757-1763, Aug. 2008.

[24] J.-C. Wu, T.-Y. Chin, S.-F. Chang, and C.-C. Chang, "2.45$\mathrm{GHz}$ CMOS reflection-type phase-shifter MMICs with minimal loss variation over quadrants of phase-shift range," IEEE Trans. Microw. Theory Techn., vol.56, no.10, pp.2180-2189, Oct. 2008.

[25] T.-Y. Chin, J.-C. Wu, S.-F. Chang, and C.-C. Chang, "Compact $\mathrm{S}-/ \mathrm{Ka}$-band CMOS quadrature hybrids with high phase balance based on multilayer transformer over-coupling technique," IEEE Trans. Microw. Theory Techn., vol.57, no.3, pp.708-715, March 2009.

[26] J. S. Park, S. Kousai, and H. Wang, "A fully differential ultracompact broadband transformer based quadrature generation scheme," Proc. Custom Integr. Circuits Conf., Sept. 2013.

[27] J. S. Park and H. Wang, "A transformer-based polyphase network for ultra-broadband quadrature signal generation," IEEE Trans. Microw. Theory Techn., vol.63, no.12, pp.4444-4457, Dec. 2015.

[28] T.-W. Li and H. Wang, "A millimeter-wave fully integrated passive reflection-type phase shifter with transformer-based multi-resonance loads for $360^{\circ}$ phase shifting," IEEE Trans.
Circuits Syst. I, vol.65, no.4, pp.1406-1419, April 2018.

[29] T.-W. Li, J. S. Park, and H. Wang, "A 2-24-GHz 360 full-span differential vector modulator phase rotator with transformer-based poly-phase quadrature network," IEEE Trans. VLSI Syst., vol.28, no.12, pp.2623-2635, Dec. 2020.

[30] R. C. Fyre, S. Kapur, and R. C. Melville, "A 2-GHz quadrature hybrid implemented in CMOS technology," IEEE J. Solid-State Circuits, vol.38, no.3, pp.550-555, March 2003.

[31] F. Ali and A. Podell, "A wide-band GaAs monolithic spiral quadrature hybrid and its circuit applications," IEEE J. SolidState Circuits, vol.26, no.10, pp.1394-1398, Oct. 1991. 